\title{
Localized biomolecular sensing enabled through plasmonic nanocavities
}

\author{
Gaël Osowiecki*, Elsie Barakat, Ali Naqavi, Hans Peter Herzig \\ Optics \& Photonics Technology Laboratory, École Polytechnique Fédérale de Lausanne (EPFL), \\ Neuchâtel, CH-2000, Switzerland
}

\begin{abstract}
We propose and study an integrated refractive index sensor which is based on a plasmonic slot cavity integrated on silicon-on-insulator wafers. In this device, the guided mode is vertically coupled to the cavity which is a metal-dielectricmetal waveguide and is separated from a photonic wire waveguide by a silicon dioxide spacer. We perform an in-depth study that links the geometrical parameters to the coupling and sensitivity. The strong coupling from the dielectric waveguide to the plasmonic slot waveguide cavity allows a local change in refractive index to be detectable with a high sensitivity of around $600 \mathrm{~nm} / \mathrm{RIU}$ in a femto-liter volume. These results are obtained with three-dimensional time domain simulations made with the CST Microwave Studio. The sensing performance of the devices are presented and compared to the practical needs to achieve localized biomolecular sensing.
\end{abstract}

Keywords: Hybrid plasmonics, Plasmon, slot waveguides, Sensors, Localized optical sensing

\section{INTRODUCTION}

At the interface of metal and dielectric, a bound electromagnetic wave can propagate over a couple of micrometers ${ }^{1}$. This type of wave is called surface plasmon polariton (SPP). In the past years, the interesting properties of SPPs have been used to advance optical sensor technology. A major property of SPPs is their ability to be confined in a smaller volume than the diffraction limit $\left(\lambda_{\text {eff }} / 2\right)$. An example of the application of the SPPs is the surface plasmon resonance (SPR) sensor ${ }^{2}$ which has been commercialized. It is used to detect the presence of molecule in a certain liquid or monitor the kinetics of the chemical reactions between different molecules without any labeling requirements ${ }^{3}$. More recently there has been a growing interest about localized surface plasmon resonance (LSPR) sensors because those sensors could compete with SPR and might be smaller and cheaper to fabricate ${ }^{4,5}$. A LSPR device is based on metallic nanoparticle aggregates or single metallic nanoparticles, which exhibit a resonance peak in the visible region of the spectrum ${ }^{6}$. By monitoring the spectral shift of this resonance peak, one can sense the refractive index changes of the surrounding medium.

Different parameters may be used to evaluate the performance of a plasmonic sensor. The mostly-used parameter is the sensitivity (S), which is a measure of the strength of light-matter interaction in the sensor. It is defined as the change of the magnitude of the sensor transduction signal in response to the change of a characteristic of the analyte, for example its refractive index. Another important parameter is the sensor Detection Limit (DL), which is defined as the ratio of the minimum detectable signal to the sensitivity. It is usually desired to increase $\mathrm{S}$ and decrease DL as far as possible. The sensitivity can be enhanced by increasing the light-matter interaction length and the detection limit can be improved by decreasing the noise of the system. For a resonator, the effective light-matter interaction length is not only determined by the sensor's physical size, but also by the resonator quality factor (Q-factor) which depends on the time that the light stays inside the resonator before leaving it. There is a trade-off between the cavity size, Q factor and wave localization.

*gael.osowiecki@epfl.ch; phone+41 216954 316; opt.epfl.ch

Biophotonics: Photonic Solutions for Better Health Care IV, edited by Jürgen Popp, Valery V. Tuchin,

Dennis L. Matthews, Francesco S. Pavone, Proc. of SPIE Vol. 9129, 91292B · ( 2014

SPIE · CCC code: $0277-786 X / 14 / \$ 18 \cdot$ doi: $10.1117 / 12.2052822$

Proc. of SPIE Vol. 9129 91292B-1 
The next milestone to achieve for refractive index sensors is the ability to detect single molecules in a sample with reasonably high concentration that allows enzymatic reactions. One way to realize this aim is to reduce the optical observation volume ${ }^{7,8}$. Having this aim in mind, here we present a theoretical study of a refractive index sensor which is based on a plasmonic slot waveguide cavity (PSWC) excited by a photonic wire waveguide sustaining a single mode. In this manuscript we search for a stronger localization than introduced in our previous work which was a PSWC array excited with a slab waveguide ${ }^{9,10}$.

We consider the scaling properties of a PSWC (size versus performance) considering the factors Q and the sensing volume. Furthermore we investigate the coupling mechanisms of light into wave-guiding nanostructures, which are related to the coupling strength and coupling length. For the simulations we use the CST Microwave Studio using either the time domain solver or the frequency domain solver which are based on Finite-Integration Technique (FIT). For mode calculations the 2D mode solver of CST MWS is used with broadband calculation for non QTEM modes enabled. We use a non-uniform hexahedral mesh with a mesh size of $2 \mathrm{~nm}$ around the metallic cavity and $\lambda / 10$ in the dielectric materials. The model contains around 10 million mesh cells and the exact number of mesh cells depends on the geometrical parameters of the device. We performed these calculations by using distributed computing.

\section{PRESENTATION OF THE PSWC SENSOR}

In this section, we present the operation principle of the PSWC sensor and the influence of its geometrical parameters on the transmitted spectrum.

\subsection{Architecture of the PSWC}

The PSWC sensor depicted in figure 1 is integrated on a SOI wafer based fabrication process with a silicon layer thickness of $220 \mathrm{~nm}$ and a buried oxide layer of $2 \mu \mathrm{m}$ thickness. Light is injected in a photonic wire waveguide made out of single crystal silicon and covered with a silicon dioxide layer of variable thickness called the "spacer" (ts). The width of the waveguide $(\mathrm{w})$ is $450 \mathrm{~nm}$ where only two propagating modes, a quasi-transverse-electric (qTE) and a quasitransverse-magnetic (qTM), are sustained at the wavelengths between $1200 \mathrm{~nm}$ and $1700 \mathrm{~nm}$. The PSWC is deposited on top of the spacer layer. It consists of two separated metallic rectangles between which a slot is formed. The slot width $(\mathrm{sw})$ is $50 \mathrm{~nm}$. The metallic layer thickness $(\mathrm{tm})$ is $20 \mathrm{~nm}$. Changing the cavity length (L) varies the position of the resonance peak in the spectrum.

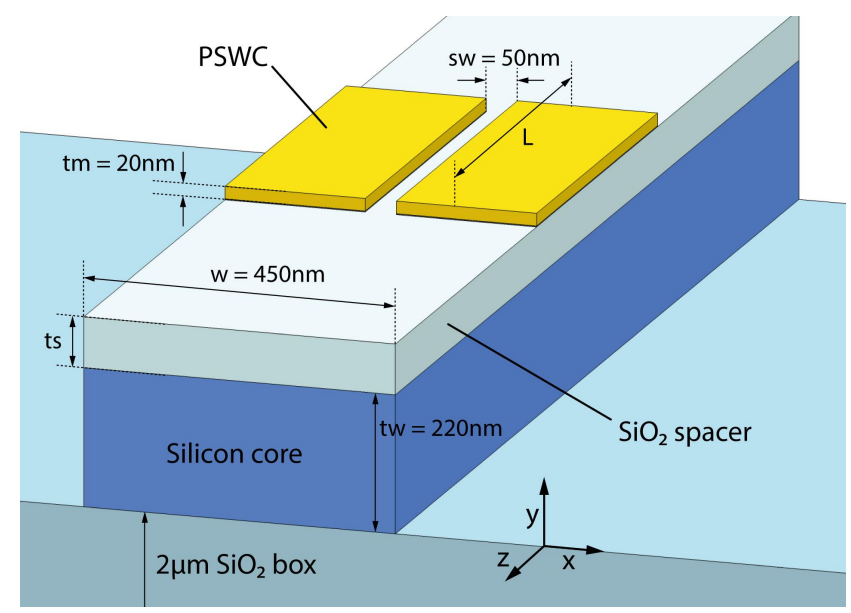

Figure 1. Sketch of the PSWC sensor with its geometrical parameters

\subsection{Operation principle}

The refractive index (RI) sensor is based on a plasmonic resonating cavity. As explained before, the plasmonic slot waveguide has the ability to confine the E-field well below the diffraction limit and to have a relatively long propagation distance ${ }^{11}$. Here we show that it is possible to use these properties to create a localized RI sensor with a detection 
volume in the order of a femto-liter. Like a Fabry-Pérot resonance between two mirrors, a truncated plasmonic slot waveguide can sustain a Fabry-Pérot resonance. We propose to excite the structure with light coming from a waveguide on an integrated platform. This allows the detection volume to be isolated from any other light sources making the sensor highly multiplexable. The refractive index change is detected by measuring the signal transmitted through the silicon wire waveguide. The transduced transmitted signal can be measured in two ways: either by measuring the spectral shift of the resonance peak or by measuring the intensity change at a single wavelength located at the inflection point of the resonance dip. In order to compare with other RI sensors, we study the sensitivity by the spectral shift of the resonance and we express it in $\mathrm{nm} /$ refractive index unit (RIU).

\subsection{Geometrical parameter choice}

For ease of use, the sensor is optimized to work around $1500 \mathrm{~nm}$. We perform simulations of the transmission spectrum as a function of two different sensor parameters: the cavity length and the spacer thickness. The 2D map in Figure 2 shows the transmission spectra as function of the cavity length for a gold PSWC with a spacer thickness of $40 \mathrm{~nm}$. The different resonance orders appear as the cavity length increases. For cavities smaller than around $200 \mathrm{~nm}$ the fundamental resonance disappears because phase matching cannot be satisfied. As the extinction ratio of the first order resonance is the most- pronounced, we use a $600 \mathrm{~nm}$ long cavity so that the resonance occurs at around $1500 \mathrm{~nm}$. The corresponding transmission curve is shown in the figure 2.

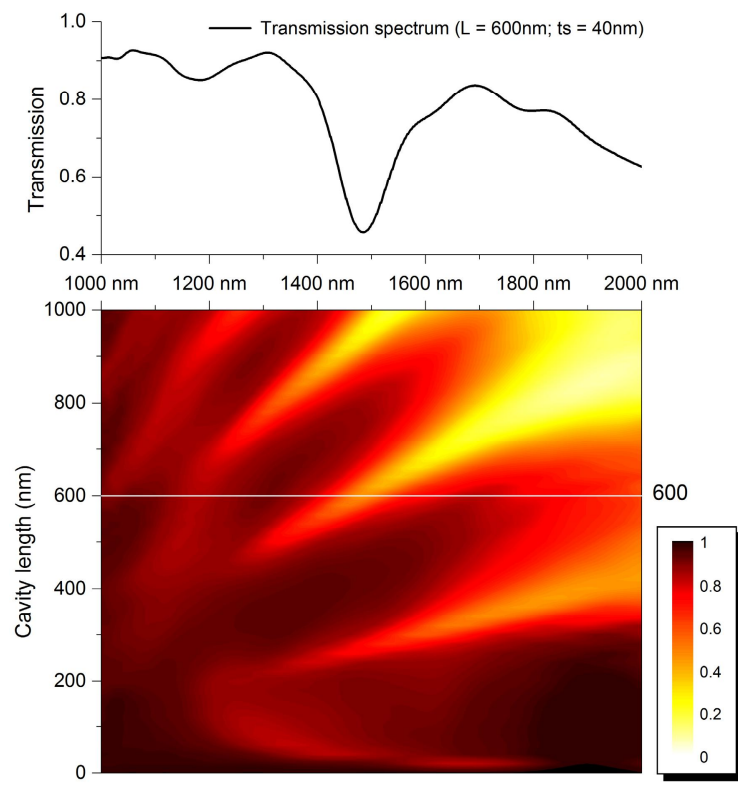

Figure 2. Collection of multiple transmission spectra of different cavity lengths of a gold PSWC with a $40 \mathrm{~nm}$ spacer thickness. The different Fabry-Pérot resonance orders can be observed as the cavity length increases. A typical transmission curve for a cavity length of $600 \mathrm{~nm}$ is shown on top.

Another important geometrical parameter is the spacer thickness which separates the silicon wire waveguide from the PSWC. It affects the coupling strength of both the plasmonic and the photonic wire waveguides. Figure 3 show that the resonance position remains constant as the spacer thickness is increased. The spacer thickness only influences the transmission in the red part of the spectrum for spacer thicknesses above $20 \mathrm{~nm}$. To work efficiently with the 1-st order harmonic resonance, we use a $40 \mathrm{~nm}$ thickness unless specified. By varying the spacer thickness, we also change its mode profile. This implies some changes in the coupling of waves in the photonic wire waveguide to the PSWC and vice-versa. We do not take into account this in the present paper. We also observe that the E-field is more confined for smaller slot width. This can be interesting to reduce the detection volume. 


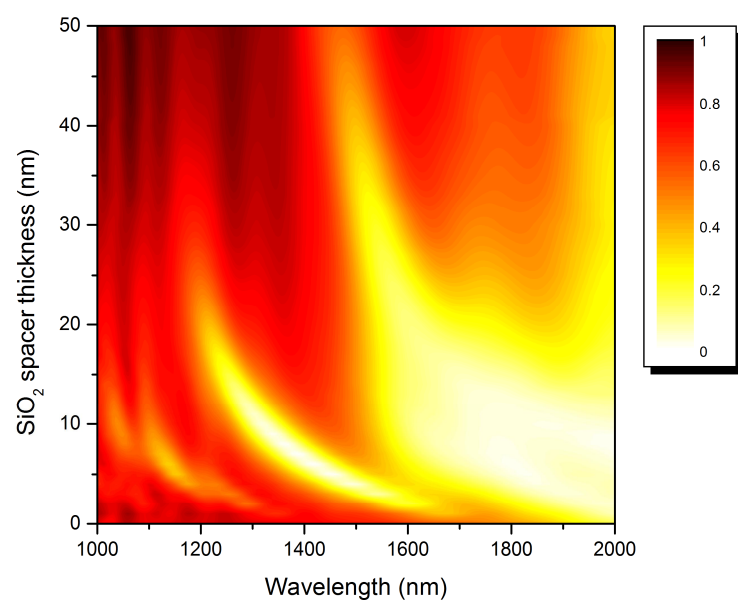

Figure 3. Collection of multiple transmission spectra of different spacer thicknesses of a PSWC having a $600 \mathrm{~nm}$ length. Again, the different Fabry-Pérot resonance orders can be observed along the spectrum.

\section{RESULTS AND DISCUSSION}

\subsection{Mode coupling}

As the excitation of the PSWC comes from the qTE mode of the photonic wire waveguide, we use a supermode approach in order to explain in an easy way the coupling mechanism ${ }^{12}$. We assume the device to operate in the weakcoupling regime. In figure 4 we show six different mode profiles by their respective E-field. The first four mode profiles are the ones from the uncoupled structures. Figure $4 \mathrm{a}$ and $4 \mathrm{~b}$ are showing the asymmetric $\left(\mathrm{A}_{0}\right)$ and symmetric $\left(\mathrm{S}_{0}\right)$ plasmonic slot waveguide modes and figure $4 \mathrm{c}$ and $4 \mathrm{~d}$ show the two qTE and qTM modes of the photonic wire waveguide. The $\mathrm{A}_{0}$ mode is highly confined in the slot between the two metal rectangles and is asymmetric with respect to the $\mathrm{y}$-axis similar to the qTE mode. Because the $\mathrm{A}_{0}$ mode has a strong overlap with the qTE mode, it should be excited more easily by it. Finally we put both structures together. The mode of the new structure can be considered as a combination of the fields of both structures. Thus we should obtain four hybrid modes. Because we are only exciting the structure with the qTE mode of the photonic wire waveguide, only two of these modes are excited. These two modes are shown on figure $4 \mathrm{e}$ and $4 \mathrm{f}$ and are called hybrid modes $\left(\mathrm{H}_{\mathrm{TE} 0} \& \mathrm{H}_{\mathrm{TE} 1}\right)$. 


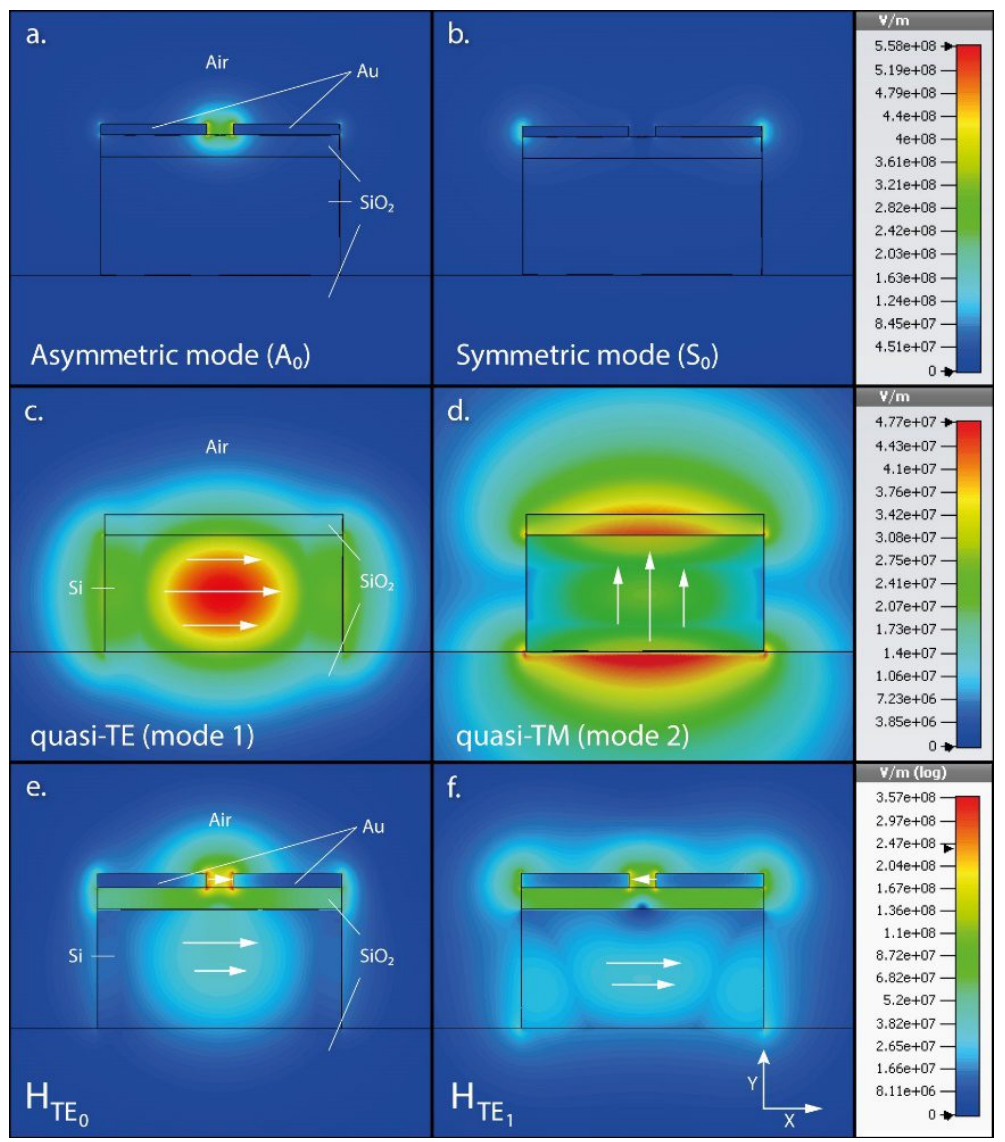

Figure 4. Magnitude of the E-field of the two asymmetric $(a, c)$ and two symmetric (b,d) uncoupled modes. (e,f) Magnitude of the E-field of the two hybrid modes resulting from the coupling of qTE, $\mathrm{A}_{0}$ and $\mathrm{S}_{0}$ modes.

We show that the coupling between the $\mathrm{H}_{\mathrm{TE} 0}$ and $\mathrm{H}_{\mathrm{TE} 1}$ mode can be described in the case of weak coupling by equation 1. The coupling length $\left(\mathrm{L}_{\mathrm{c}}\right)$ shows the length that is required for the energy to be transferred from one waveguide to the other one, and is defined as.

$$
\mathrm{L}_{\mathrm{c}}=\frac{\pi}{\beta_{\text {even }}-\beta_{\text {odd }}}
$$

The two modes excited in the coupled guiding structure interfere with each other because their propagation constant is different. When this difference is small as in an integrated photonic wire waveguide directional coupler the coupling length becomes large (i.e. in the order of $10-20 \mu \mathrm{m}$ ) ${ }^{13}$. In our case we can achieve very small coupling length of around $1.75 \mu \mathrm{m}$. 


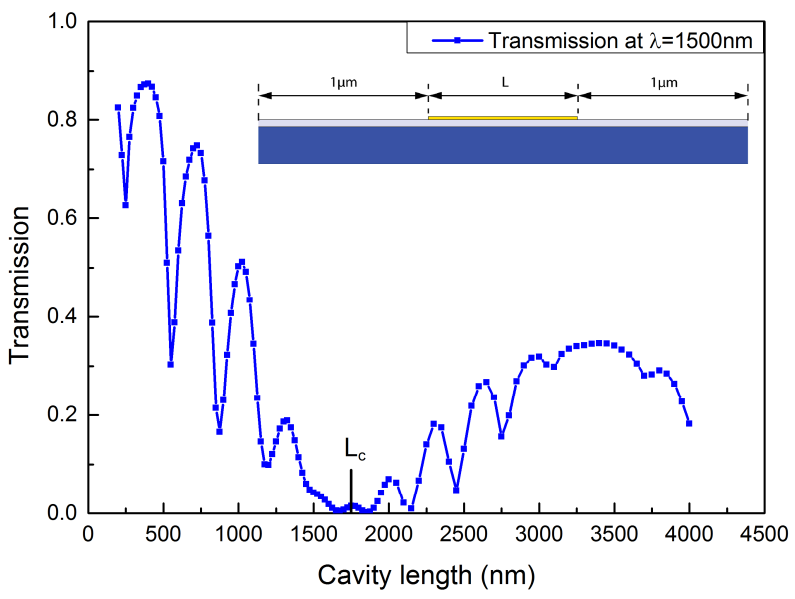

Figure 5. Transmission at $\lambda=1500 \mathrm{~nm}$ of a gold PSWC (ts $=40 \mathrm{~nm})$ versus cavity length showing the Fabry-Pérot response combined with the low frequency coupling frequency.

Figure 5 shows the transmission of the PSWC at $\lambda=1500 \mathrm{~nm}$ for different cavity lengths calculated by using the frequency domain solver of CST MWS. Figure 5 shows the transmission as a function of the cavity length. The cavity length of $1.75 \mu \mathrm{m}$ corresponds to the coupling length. At this cavity length, the energy is completely located in the PSWC, thus light is no longer transmitted through the silicon wire waveguide and the transmission drops to 0 . In that case, most of the light is scattered out at the end of the PSWC. A PSWC of $600 \mathrm{~nm}$ length is much shorter than its actual coupling length. The coupling length increases for higher spacer thicknesses. To use this architecture as a dielectricplasmonic coupler, a smaller spacer can allow fabrication of a more compact device. In the next section we link the coupling length to the field enhancement.

\subsection{Field enhancement and sensitivity}

The PSWC works like a highly spatially localized refractive index sensor with high sensitivity thanks to its ability to strongly enhance the light intensity inside its slot. We present simulations that show the normalized near-field enhancement at the resonance position. Figure 6 demonstrates a top view of the normalized field enhancement of the PSWC, excited from the photonic wire waveguide coming from the bottom. The E-field is locally enhanced up to 8000 times compared to the case of a simple photonics wire waveguide. Furthermore this strong field enhancement originates from the Fabry-Pérot resonance and the enhancement drops drastically for off-resonance wavelengths. For higher order resonances at the same wavelength (i.e. longer cavity length) the average field enhancement over the whole cavity increases because the cavity length approaches the coupling length.

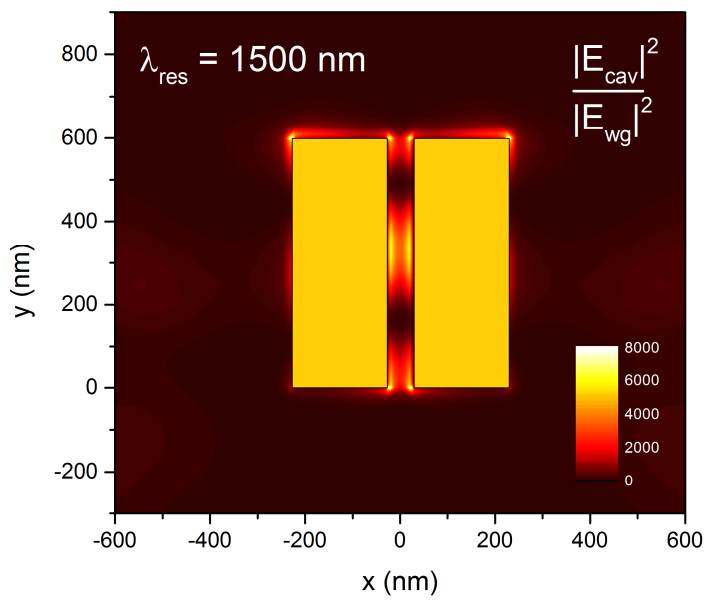

Figure 6. Normalized E-field enhancement on a plane passing through the middle of the PSWC (excitation from bottom). 
Finally we show the simulation results of the bulk sensitivity to refractive index changes of the PSWC sensor for three different metals as function of the spacer thickness. It is calculated by measuring the shift of the resonance peak in which the outer medium refractive index has been changed between water and acetone. Figure 7 shows the bulk sensitivity for $600 \mathrm{~nm}$ long PSWCs made out of gold, silver and aluminium. The dielectric constants were taken form Johnson \& Christy ${ }^{14}$ for all three metals and fitted by an n-th order polynomial to be used equivalently in time domain and in frequency domain. The bulk sensitivity rises with the spacer thickness. A peak sensitivity of $625 \mathrm{~nm} / \mathrm{RIU}$ is obtained for aluminium at a spacer thickness of $120 \mathrm{~nm}$. Because of its high optical losses, the extinction ratio of the resonance is weak for aluminium. Thus, it becomes difficult to experimentally measure the resonance shift that lowers the detection limit of the sensor. The best quality factor is obtained with silver, thanks to its low optical losses $(\mathrm{Q} \approx 12)$. Gold sensitivity is between the two others metals.

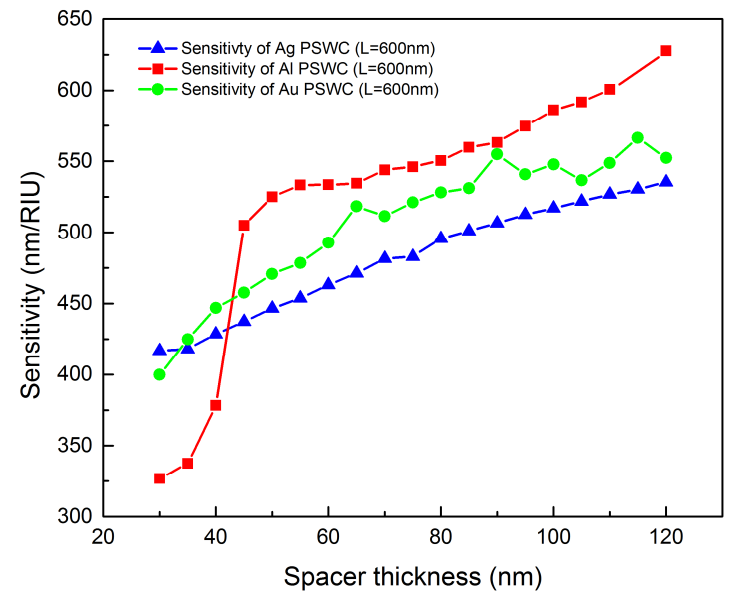

Figure 7. Sensitivity comparison between PSWC using three different metals

This theoretical study of the general behavior of the PSWC sensor provides an understanding of the relation between sensitivity and coupling. It can be used as a guide for the fabrication and characterization of the sensor. This type of architecture which lies in between LSPR and SPR can be of great use in localized biosensing at high concentrations.

\section{CONCLUSION}

We showed a numerical study of the plasmonic slot waveguide cavity which can be used as a localized refractive index sensor in the near-infrared. Thanks to the strong confinement of the light in the slot, it can measure a local refractive index change, which can be for example introduced by a layer of proteins. Our sensor architecture bridges the gap between SPR and LSPR technics. It has the advantage to be highly multiplexable. It is also possible to integrate multiple PSWC with resonance peak positions distributed over the spectrum to monitor the local changes of refractive index over a millimeter range length scale. We further explained the coupling mechanism between the photonic wire waveguide and the plasmonic slot waveguide. Strong near-field enhancement was achieved in the Fabry-Pérot like PSWC, which can be exploited for fluorescence detection on labeled protein or Raman scattering. We demonstrated that the PSWC sensor could reach sensitivities up to $625 \mathrm{~nm} / \mathrm{RIU}$ for a detection volume of a femto-liter which is promising to approach the high concentration limit in molecular biosensing.

\section{REFERENCES}

[1] Schuller, J. A., Barnard, E. S., Cai, W., Jun, Y. C., White, J. S.., Brongersma, M. L., "Plasmonics for extreme light concentration and manipulation," Nat. Mater. 9(3), 193-204 (2010).

[2] Homola, J., Yee, S. S.., Gauglitz, G., "Surface plasmon resonance sensors: review," Sens. Actuators B Chem. 54(1-2), 3-15 (1999).

[3] Homola, J.., Dostálek, J., Surface plasmon resonance based sensors, Springer (2006). 
[4] Chen, Y.., Ming, H., "Review of surface plasmon resonance and localized surface plasmon resonance sensor," Photonic Sens. 2(1), 37-49 (2012).

[5] Willets, K. A.., Van Duyne, R. P., "Localized Surface Plasmon Resonance Spectroscopy and Sensing," Annu. Rev. Phys. Chem. 58(1), 267-297 (2007).

[6] Mayer, K. M.., Hafner, J. H., "Localized Surface Plasmon Resonance Sensors," Chem. Rev. 111(6), 3828-3857 (2011).

[7] Holzmeister, P., Acuna, G. P., Grohmann, D.., Tinnefeld, P., "Breaking the concentration limit of optical singlemolecule detection," Chem. Soc. Rev. 43(4), 1014-1028 (2013).

[8] Luo, Y., Chamanzar, M.., Adibi, A., "Compact on-chip plasmonic light concentration based on a hybrid photonic-plasmonic structure," Opt. Express 21(2), 1898-1910 (2013).

[9] Cosentino, A., Tan, Q., Roussey, M.., Herzig, H. P., "Refractive index sensor based on slot waveguide cavity,” J Eur. Opt Soc Rap Public 7, 12039 (2012).

[10] Tan, Q., Cosentino, A., Roussey, M.., Herzig, H. P., "Theoretical and experimental study of a 30nm metallic slot array," J. Opt. Soc. Am. B 28(7), 1711-1715 (2011).

[11] Veronis, G.., Fan, S., "Modes of Subwavelength Plasmonic Slot Waveguides," J. Light. Technol. 25(9), 2511 2521 (2007).

[12] Berini, P., "Plasmon-polariton waves guided by thin lossy metal films of finite width: Bound modes of asymmetric structures," Phys. Rev. B 63(12), 125417 (2001).

[13] Cong, G. W., Suzuki, K., Kim, S. H., Tanizawa, K., Namiki, S.., Kawashima, H., "Demonstration of a 3-dB directional coupler with enhanced robustness to gap variations for silicon wire waveguides," Opt. Express 22(2), 2051-2059 (2014).

[14] Johnson, P. B.., Christy, R.-W., "Optical constants of the noble metals," Phys. Rev. B 6(12), 4370 (1972). 